\title{
FC06-03
}

\section{CONDITIONAL ACCEPTANCE AS A DISTINCT FEATURE OF SOCIALLY-PRESCRIBED PERFECTIONISM: A STUDY IN}

PORTUGUESE PREGNANT WOMEN

A. Macedo ${ }^{1}$, S. Carvalho Bos ${ }^{1}$, B. Maia ${ }^{1}$, M.J. Soares ${ }^{1}$, M. Marques ${ }^{1}$, A.T. Pereira ${ }^{1}$, A. Allen Gomes ${ }^{2}$, J. Valente ${ }^{1}$, M.H. Azevedo

${ }^{1}$ Institute of Medical Psychology, Faculty of Medicine, Coimbra University, Coimbra, ${ }^{2}$ Department of Educational Sciences, Aveiro University, Aveiro, Portugal

Aim: To investigate associations between perfectionism dimensions and psychological distress 421 pregnant women ( $M=29.8$, $\mathrm{SD}=4.48$ years) completed measures of Self-Oriented Perfectionism (SOP), Socially-Prescribed Perfectionism (SPP) (MPS; Hewitt \& Flett, 1991; Soares et al., 2003) , mood (POMS; McNair et al., 1971; Azevedo et al., 1991) and depressive symptomatology (BDIII; Beck et al., 1996; Coelho et al., 2002).

Methods: A 2-factor model of Perfectionism with SOP and SPP dimensions and a 3-factor model with SOP, SPP-Others' High Standards and SPP-Conditional Acceptance factors were explored. Correlations and Linear Regressions were calculated between perfectionism factors and mood variables/depressive symptoms.

Results: Higher levels of SPP factors were in general associated with increased Anxiety, Depression, Anger, Fatigue and Confusion, with decreased Vigour and with more severe depressive symptomatology. SPP dimension and both SPP sub-scales explained depressive symptoms.

Conclusion: Our results, in contrast with those from the study of Campbell and DiPaula (2002) did not confirm a preferential association between SPP-Conditional Acceptance and psychological distress (PD), revealing that both components of SPP were associated with PD. 